\title{
The Relationship of Blood Glucose and Blood Pressure In Age-Related Cataracts
}

\author{
Duong Dieu* \\ Department of Ophthalmology, Angiang Provincial Hospital, Vietnam
}

Received: October 31,2017; Published: November 06, 2017

*Corresponding author: Duong Dieu, Department of Ophthalmology, Faculty of Medicine- Nguyen Tat Thanh University, Angiang Provincial Hospital, Add: 300A Nguyen Tat Thanh Street- Dist. 4- Hochiminh City, Vietnam, Tel: +84918213354 ; Email: duongdieumd@gmail.com, duongdieumd@ntt.edu.vn

\section{Introduction}

Surgical correction of cataracts now has a high success rate and results in restored sight for affected patients. Researchers, however, continue to try and find ways to prevent or delay development of the condition. The ability to delay the onset of cataract by 10 years would eliminate the need for one-half of all cataract surgeries. This paper discusses the relationship of blood glucose and blood pressure to the development of age-related cataracts. Cataract is defined as opacity of the crystalline lens, and development is influenced by a number of exogenous and endogenous factors. The greatest risk factor is age. Diabetes mellitus is also associated with the development of cortical and posterior sub capsular cataract [1]. The Beaver Dam Eye Study and the Cataract Patient Outcomes Research Team have reported an association between age-related cataracts and systemic cardiovascular or atherosclerotic disease [2]. Debate continues, however, with regard to the exact causes of cataractogenesis and ways in which it may be prevented [1].

\section{Diabetes}

Several studies have reported an association between glucose and the increased risk of cataract formation in animals and humans [3]. In diabetics, alternative mechanisms may be superimposed on the normal aging process [4] oxidative stress and glucose metabolism is principal mechanisms of cataract formation [5]. The Beaver Dam Eye Study reported that in diabetes mellitus, over a period of 5 years, there was an increased incidence of cortical and posterior sub capsular cataract and progression of more minor cortical and posterior sub capsular lens opacities. These changes may be related to levels of glycaemia [6]. Retinopathy affects 24 to $70 \%$ of type 2 diabetics while 18 to $22 \%$ will develop cataracts: neuro-ophthalmological complications are rare [7]. Diabetics have up to a 12-fold increased risk of cataract formation compared with non-diabetics [5]. In a Vietnamese long-term follow-up study, 50\% of type 2 diabetics developed cataracts [8]. Mature dense cataracts are rare but, when they occur, do not appear to resolve with control of hyperglycemia [9]. Surgical removal is thus required. The report of a 9-year old girl who presented with rapidly developing bilateral mature cataracts adds support to the theory that metabolic factors are associated with cataractogenesis [10].

It has been suggested that glycosylation of the lens proteins leads to the development of permanent lens opacities. According to one researcher, oxidative stress and glucid stress are responsible for cataract formation. It is hoped that a new drug for fighting aldose reductase activity will be available in human [5,11]. Datta et al. [12] offered a new hypothesis that was conducted by linking a clinical observation with evidence from experimental animal models. Biochemically, the highly conserved and inducible enzyme aldose reductase was implicated in the development of these cataracts with possible contributions from glycation and oxidative stress [13]. In the management of such cataracts, a larger than normal anterior capsulotomy is recommended at the time of cataract surgery to permit better visualization of the fundus for future management of diabetic retinopathy. Both of the aforementioned studies suggest that cataract extraction and intraocular lens implantation with small incision phacoemulsification surgery is no longer a contraindication and may be of great benefit to patients with advanced diabetic ocular disease [14].

\section{Cardiovascular Disease}

The Framingham Eye Study suggested an association between cataract and a number of cardiovascular risk factors. The latter included systemic hypertension, diabetes mellitus and elevated serum phospholipid levels. The Beaver Dam Eye Study suggested that serum lipids, glycated haemoglobin and oxidative stress may play a role in cataractogenesis [15]. Cardiovascular disease and associated risk factors were not thought to affect the development of age-related cataract [2]. A weak association between atherosclerosis-related morbidity and visually significant cataract has been reported and is strongest for people aged 65 to 69 years [11]. As the prevalence of atherosclerosis and cataract increases with age, it was rationalized that a biological association between the two may be observed in younger individuals. 
The presence of such an association has raised the question of whether a deficiency in the natural defenses against free radicals contributes to the development of both cataracts and atherosclerosis [11].

Various dietary antioxidants such as ascorbic acid, vitamin E, and beta-carotene, have been examined for their possible roles in preventing damage at cellular DNA, lipid, and proteins. Oxidative stress, resulting of the information of lipid peroxides, has been suggested to contribute to pathologic processes involved in acing and systemic diseases such as atherosclerosis, diabetes, and chronic renal failure [16].

\section{Hypertension and Hyperglycaemia}

High blood pressure is a late complication of diabetes. The control of hyperglycemia is also much poorer in hypertensive diabetics. Datta et al. [12] offered a new hypothesis regarding the poorly understood pathogenesis of these metabolic cataracts in type I diabetic patients [12]. A high level of glycosylated haemoglobin $\mathrm{HbA1c}$, prolonged duration of hyperglycemia prior to diagnosis, an adolescent age group and preponderance of females were all noted to be significant [12]. The Beaver Dam Eye Study has found significant relationships between glycaemia and the incidence of nuclear and cortical cataracts in people with olderonset diabetes, and has found little evidence of an association between cardiovascular disease and its risk factors to the incidence of cataracts. These changes may be related to the level of glycaemia [6].

\section{Hypertension and Blood Glucose in Cataract Patients}

The relationship between a limited level of blood glucose (BG) and high blood pressure (BP) in age-related cataracts in a crosssectional case-control study in Vietnam [13] showed an increasing blood glucose and blood pressure with the age groups 50s, $60 \mathrm{~s}$ and 70s between cataracts and non-cataracts. A Japanese study evaluated blood pressure, intraocular pressure and body mass index in office workers aged 20 to 79 years of age. Intraocular pressure decreased with age in a cross-sectional analysis but increased with age with a longitudinal analysis, in both men and women. Blood pressure was positively correlated to intraocular pressure.14 showed an increasing of blood pressure with age. (In this large Japanese population study of age-related changed in intraocular pressure, did not study on cataract)

The relationship of BG $\otimes 6.1 \mathrm{mmol} / \mathrm{L}$ with $\mathrm{BP} \otimes 140 / 90 \mathrm{mmHg}$ between the cataract and non-cataract groups was significantly different $(\mathrm{OR}=2.1$ [1-4.4]; $\mathrm{p}=0.04) . \mathrm{BG}$ at a single point in time did not accurately reflect the cataract patients' exposure [13]. Up to now we do not have any information on this relationship. This finding may have direct use in prevention. General practitioners can detect early onset retinopathy as well as cataracts and act by manipulating risk factors [13].

\section{Conclusion}

Hypertension and a high level of glycosylated haemoglobin have particularly been associated with cataract formation. Future studies may reveal the exact nature of this relationship. It has been proposed that cataract surgery is not a risk factor for the progression of diabetic retinopathy and that diabetic patients may benefit from safe cataract extraction by phacoemulsification with minimal complications.

\section{References}

1. Hutnik CM, Nichols BD (1998) Cataracts in systemic diseases and syndromes. Curr Opin Ophthalmol 9(1): 14-19.

2. Hutnik CM, Nichols BD (1999) Cataracts in systemic diseases and syndromes. Curr Opin Ophthalmol 10: 22-28.

3. Donnelly CA, Seth J, Clayton RM, Phillips CI, Cuthbert J, et al. (1995) Some blood plasma constituents correlate with human cataract. Br J Ophthalmol 79(11): 1036-1041.

4. Perira PC, Ramatho J, Sfaro CJ, Mota MC (1994) Age-related changes in normal and cataractous human lens crystalline, separated by fastperformance liquid chromatography. Ophthalmic Res 26: 149-157.

5. Kinoshita JH (1992) Les atteintes criatalliniennes chez le diabetique. Cataractes 4: 7-9.

6. Klein BE, Klein R, Lee KE (1998) Diabetes, cardiovascular disease, selected cardiovascular disease risk factors, and the 5-year incidence of age-related cataract and progression of lens opacities: the Beaver Dam Eye Study. Am J Ophthalmol 126(6): 782-790.

7. Aziz T (1996) Incidence of ophthalmic problems in diabetic patients in Pakistan: a preliminary report. Ophthalmic Practice 2: 4-6.

8. Ngo Nhu Hoa (1988) Some considerations on the epidemiology of cataract in Ho Chi Minh. Ophthalmic Information of HCM City, Vietnam 72-73.

9. Cornwell M, Lepre F (1995) Acute irreversible cataracts in diabetes mellitus. Austr NZ J Ophthalmol 23(3): 221-223.

10. Santiago AP, Rosenbaum AL, Masket S (1997) Insulin-dependent diabetes mellitus appearing as bilateral mature diabetic cataracts in a child. Arch Ophthalmol 115(3): 422-423.

11. Street DA, Javitt C, Wang Q, Tielsch JM, Canner JK, et al. (1996) Atherosclerotic disease in patients undergoing cataract extraction: a nationwide case-control study. Arch Ophthal 114(11): 1407-1411.

12. Datta V, Swift PG, Woodruff GH, Harris RF (1997) Metabolic cataracts in newly diagnosed diabetes. Arch Dis Child 76(2): 118-120.

13. Duong Dieu, Le Vi Hung, Tran Nguyet Thanh (1998) Epidemiology of cataract: The relationship of blood glucose and blood pressure in cataract. Researches of Hanoi Medical College, Vietnam 4: 28-29.

14. Nomura H, Shimokata H, Ando F, Miyake Y, Kuzuya F (1999) Age-related changes in intraocular pressure in a large Japanese population: a crosssectional and longitudinal study. Ophthalmol 106(10): 2016-2022.

15. Honjo M Ogura Y (1998) surgical results of pars plana victrectomy combined with phacoemulsification and intraocular lens implantation for complications of proliferative diabetic retinopathy. Ophthalmic Surg Lasers 29(2): 99-105.

16. Babizhayev MA (1996) Failure to withstand oxidative stress induced by phospholipid hydroperoxides as a possible cause of the lens opacities in systemic diseases and ageing. Biochim Biophys Acta 1315(2): 87-99. 


$\begin{array}{ll}\text { BIOMEDICAL } & \text { Assets of Publishing with us } \\ \text { RESEARCHES } & \text { Global archiving of articles } \\ \text { - Immediate, unrestricted online access } & \text { - Rigorous Peer Review Process } \\ & \text { - Authors Retain Copyrights }\end{array}$

